\title{
Using Time-to-React based on Naturalistic Traffic Object Behavior for Scenario-Based Risk Assessment of Automated Driving
}

\author{
Sebastian Wagner, Korbinian Groh, Thomas Kühbeck, Michael Dörfel, Alois Knoll
}

\begin{abstract}
The steady improvement of advanced driving assistance systems (ADAS) and the leap towards automated driving (AD) require novel methods for assessing the safety of those, which is a major subject for current research. Different proposals cope with the massive testing effort to assure the safety of such systems. These proposals include virtualization of testing, usage of stochastic methods and reduction of the necessary real world driving tests. Despite these different approaches, they all rely on the same basis: The behavior assessment of the vehicle under test, which results in a measurement of risk.

This paper presents a novel approach to measure the criticality of a given driving scenario fitted on the requirements of testing. A Monte-Carlo simulation, which uses the input of a motion prediction model as variation parameters, determines the possible evolutions of a scenario at every time step. The distributions of these parameters have been fitted to data obtained by a large-scale field tests. These evolutions are then analyzed individually by considering the Time-To-React (TTR) measure. Finally a single value of accident risk between 0 and 1 can be assigned to the scenario.

Index Terms-Autonomous vehicles, Vehicle safety, Risk analysis, Performance analysis
\end{abstract}

\section{INTRODUCTION}

Due to the fast emerging field of automated vehicle development, the automated assessment of driving scenes became the interest of current research. Literature agrees, that prior to the release of automated driving systems to the market, a vast amount of testing needs to be conducted [1]. Apart from efforts of reducing the quantity of tests [2]-[5], it is also necessary to evaluate the performance of the automated function in those tests. A binary classification of situations into accident and accident-free would certainly lack relevant information. A near miss affiliated to no accident would undoubtedly lead to the passenger's rejection of the automated driving function for example. A continuous measure of a scenario's criticality is required.

Within automated driving systems, such assessment metrics of scenarios are a key element of the decision making [6]-[10]. However, those are designed to be calculated in real-time on resource limited embedded systems and hence include simplifications due to computational restrictions. This paper considers a different use case: For testing purposes a driving situation can be assessed afterwards and therefore

S. Wagner and A. Knoll are with Robotics and Embedded Systems, Department of Informatics, Technische Universität München, Munich, Germany

K. Groh, T. Kühbeck and M. Dörfel are with the BMW AG, Munich, Germany offline in post-processing on a pc or cluster, overcoming computational limits.

Furthermore, the requirements on a risk assessment for safety evaluation purposes differ from those for algorithms used within automated driving vehicles. First of all, a measure indicating safety for testing should be free of false negatives, i.e. a scene that is dangerous in reality is underestimated by the method (Table I). False negative classification would lead to the fact that a faulty behavior of the automated driving function could not be recognized. An existing approach to fulfill this requirement performs a worst-case estimation of the scene [11]. Herein, the time to collision (TTC) is estimated under the assumption that all traffic participants try to achieve an accident as fast as possible. However, this approach reveals a lot of false positives by labeling harmless scenes as risky, since the prior assumption is certainly not true. The function would be rejected in some cases despite adequate behavior. For these reasons a slight overestimation of risk is desired. A second requirement on testing is its deterministic results. If the test can be repeated with identical results, critical behavior of the AD system can be debugged easier.

TABLE I

FALSE-Positives AND FALSE-Negatives

\begin{tabular}{|c|c||c|c|}
\hline \multicolumn{2}{|c||}{} & \multicolumn{2}{c|}{ real risk } \\
\cline { 3 - 4 } \multicolumn{2}{|c|}{} & low & high \\
\hline \hline \multirow{3}{*}{$\begin{array}{c}\text { estimated } \\
\text { risk }\end{array}$} & low & $\begin{array}{c}\text { true } \\
\text { negative }\end{array}$ & $\begin{array}{c}\text { false } \\
\text { negative }\end{array}$ \\
\cline { 2 - 4 } & high & $\begin{array}{c}\text { false } \\
\text { positive }\end{array}$ & $\begin{array}{c}\text { true } \\
\text { positive }\end{array}$ \\
\hline
\end{tabular}

Hence, this paper presents a novel approach that considers multiple possible scene evolutions through Monte-Carlo simulation together with their probabilities of occurrence. Additionally, time-to react (TTR) is applied as a superior alternative to TTC for assessing the criticality of a single possible collision [12].

Common approaches to risk assessment are done in two steps [11], [13]-[15]:

1) Predicting future evolution of traffic objects (TOs) in a scene

2) Using the gathered information to generate a measure of risk 


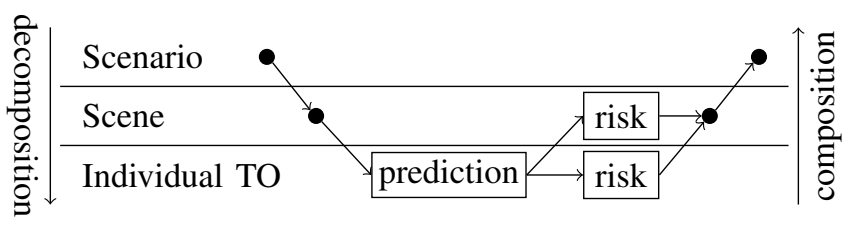

Fig. 1. Conceptual flow of the proposed risk assessment method through the abstraction layers of a scenario defined by Ulbrich [16].

In this context a scene is a certain snapshot in time of a scenario or test drive based on the definitions of Ulbrich [16]. These layers of abstraction can be seen in Fig. 1. The prediction step is covered in section II. For evaluating the risk in that scene, this work uses possible collisions between the vehicle under test (EGO) with all TOs to define a TTRbased value of criticality or risk. Two calculation methods, one conducted on TO level and one on scene level are presented in section III. One can get the risk measure for a whole scenario by computing the maximum scene risk afterwards. Followed by tests on representative scenarios and experimental results in section IV. The last section $\mathrm{V}$ concludes the findings and suggests possible future work within this area of research.

\section{Monte-Carlo Scene Prediction}

The prediction of the scene generates knowledge for possible outcomes within the near future. Some approaches calculate only the most likely outcome of a scene [12], [17], [18]. Despite being computationally effective, it ignores other possible scene evolutions and hence is not suitable for this approach. A prediction of as many future outcomes as possible is necessary and can be achieved by either MonteCarlo simulation [14], [15] or the computation of reachable sets in the form of a discretized vehicle state space [10], [19]. The latter is much faster but suffers from additional errors due to the discretization [10]. Hence, Monte-Carlo simulation is used for the presented approach. Therefore, the used model for traffic object simulation is introduced first.

\section{A. Traffic Object Models}

The models of traffic objects like vehicles can generally be categorized into three types [13]. Physics-based models follow movement equations the kinematic model of a vehicle. Maneuver-based models consider the traffic environment for example information about the road structure. The most complex category additionally includes interactions between all traffic participants. In their order of appearance, the models become valid for a longer horizon of prediction, but also demand more computational resources and are more difficult to implement. In contrast to online assessment methods supporting decision making of the AD function, a shorter period of prediction is sufficient for a post-processing method. Farther predictions reveal possible risky situations earlier. However, in post-processing the future is known and this situation is revealed during the assessment of subsequent scenes. Hence the prediction is only performed as far as possible collisions are considered relevant and a physics based model is sufficient.

For short-term-prediction a simple constant turn rate and acceleration (CTRA) model is defined. Let $x_{1}(t), x_{2}(t)$ and $\psi(t)$ define the Cartesian position of the vehicle in the world and its orientation towards the $x_{1}$-axis at a specific time $t$ respectively, while its velocity in the direction of the heading is $v(t)$. Furthermore the inputs to the system are the acceleration along the vehicles heading $a$ and the turn rate $\omega$. The CTRA model is defined by the following set of differential equations [14]:

$$
\begin{aligned}
\dot{x}_{1}(t) & =v(t) \cdot \cos (\psi(t)) \\
\dot{x}_{2}(t) & =v(t) \cdot \sin (\psi(t)) \\
\dot{\psi}(t) & =\omega \\
\dot{v}(t) & =a
\end{aligned}
$$

The constrained movement defined by those equations is suitable for objects of the type car, truck or motorcycle. Other traffic partners such as pedestrians require different formulas but are omitted as the scope of this paper asses the risk between motor vehicles.

\section{B. Scene Prediction}

As mentioned above, a set of possible scene outcomes is predicted using Monte-Carlo simulation on the CTRA model (1). The dimensions of variation are the inputs $a$ and $\omega$ for the vehicle model. At the current time step $t_{0}$ the inputs $a_{0}$ and $\omega_{0}$ are known. It is a high probability that a human driver keeps these inputs for a short period of time. However, deviations from these are also possible. The farther away from current inputs, the less likely they are. Hence Gaussian distributions for future inputs based the current inputs are modeled.

In order to keep in contact with reality, the shapes of these distributions are derived from recordings of BMW vehicles from the naturalistic driving study called euroFOT [20]. The target is to model a Gaussian distributions $f_{a}\left(a \mid v_{0}, a_{0}\right)$ for future inputs of $a$ depending on the current input $a_{0}=a\left(t_{0}\right)$ and velocity $v_{0}=v\left(t_{0}\right)$ :

$$
f_{a}\left(a \mid v_{0}, a_{0}\right)=\frac{1}{\sigma_{a}\left(v_{0}, a_{0}\right) \sqrt{2 \pi}} e^{-\frac{1}{2}\left(\frac{a-\mu_{a}\left(v_{0}, a_{0}\right)}{\sigma_{a}\left(v_{0}, a_{0}\right)}\right)^{2}} .
$$

Hence the parameters mean $\mu_{a}\left(v_{0}, a_{0}\right)$ and standard deviation $\sigma_{a}\left(v_{0}, a_{0}\right)$ are to be determined. For this purpose the euroFOT dataset is parsed for all situations that are within a margin around $v_{0}$ and $a_{0}$., which results in $N_{\mathrm{pt}}$ extracted situations. From the found occasion the acceleration $a(n)\left(n \in\left\{1, \ldots, N_{\mathrm{pt}}\right\}\right)$ in the near future is observed. Particularly the average turn rate over the next second is equal to the change of $\psi(t)$ due to the design of the model (1) and reveals a Gaussian distributed sample set for fitting the curve from (2). Then the maximum of the logarithmic likelihood function

$$
\mathcal{L}\left(a, \mu_{a}, \sigma_{a}\right)=-\frac{N_{\mathrm{pt}}}{2} \log \left(2 \pi \sigma_{a}^{2}\right)-\sum_{n=1}^{N_{\mathrm{pt}}} \frac{\left(a(n)-\mu_{a}\right)^{2}}{2 \sigma_{a}^{2}},
$$




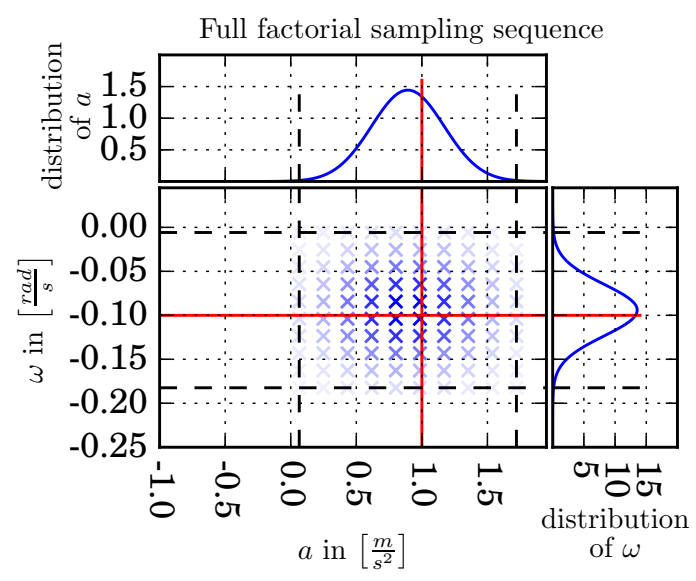

Fig. 2. Exemplary distribution of possible model inputs $a$ and $\omega$ around the actual inputs $a_{0}$ and $\omega_{0}$ indicated by red lines.

reveals the desired parameters. Repeating the process for a wide range of $v_{0}$ and $a_{0}$ results in parameter surfaces for $\mu_{a}$ and $\sigma_{a}$ over $v_{0}$ and $a_{0}$. The distribution $f_{\omega}\left(\omega \mid v_{0}, \omega_{0}\right)$ is defined and derived analogously.

An exemplary distribution for $a$ and $\omega$ is shown in Fig. 2 for the current inputs $a_{0}=1 \frac{\mathrm{m}}{\mathrm{s}^{2}}$ and $\omega_{0}=-0.1 \frac{\mathrm{rad}}{\mathrm{s}}$ indicated by red lines. The top and right plots show the just derived distribution functions $f_{a}$ and $f_{\omega}$ respectively. The distributions form around the actual inputs and verify the claim that the driver most likely will keep the inputs stable for a short period of time. A small deviation of the distribution centers towards the actual inputs can be noticed. This is due to the natural behavior of drivers which tend to return to neutral driving. The sampling area in the main plot is constrained by the $3 \sigma$-interval around the median. Thus $99.73 \%$ of the possible inputs are covered in both directions. Within this area are $N_{\mathrm{smp}}=100$ points from the set $\mathcal{V}$ uniformly distributed through the full factorial design fading out with decreasing probability of occurrence. This joint probability $f$ is calculated through

$$
f\left(a, \omega, v_{0}, a_{0}, \omega_{0}\right)=\frac{f_{a}\left(a \mid v_{0}, a_{0}\right) \cdot f_{\omega}\left(\omega \mid v_{0}, \omega_{0}\right)}{\sum_{\{a, \omega\} \in \mathcal{V}} f_{a}\left(a \mid v_{0}, a_{0}\right) \cdot f_{\omega}\left(\omega \mid v_{0}, \omega_{0}\right)} .
$$

Observing the distribution parameters over a wide range of actual inputs reveals that the distributions are the narrowest at zero inputs or neutral driving and widen up with actual inputs farther away from zero. This implies a neutral driver is more likely to stay neutral while one in a more dynamic situation is more likely to change the inputs. The standard deviation also decreases with increasing velocity presumably to the increasing limitation of vehicle dynamics at high velocities.

The sampled inputs are now used to simulate $N_{\text {smp }}=100$ trajectories as possible future evolutions of the CTRA model. For the exemplary case with actual inputs $a_{0}=1 \frac{\mathrm{m}}{\mathrm{s}^{2}}$ and $\omega_{0}=-0.1 \frac{\mathrm{rad}}{\mathrm{s}}$, the results are shown in Fig. 3. The initial state used in this example is a vehicle driving $14 \frac{\mathrm{m}}{\mathrm{s}}$ which is currently located at $x_{1}=0 \mathrm{~m}, x_{2}=3.5 \mathrm{~m}$ and has a heading along the $x_{1}$-axis. The prediction horizon is chosen

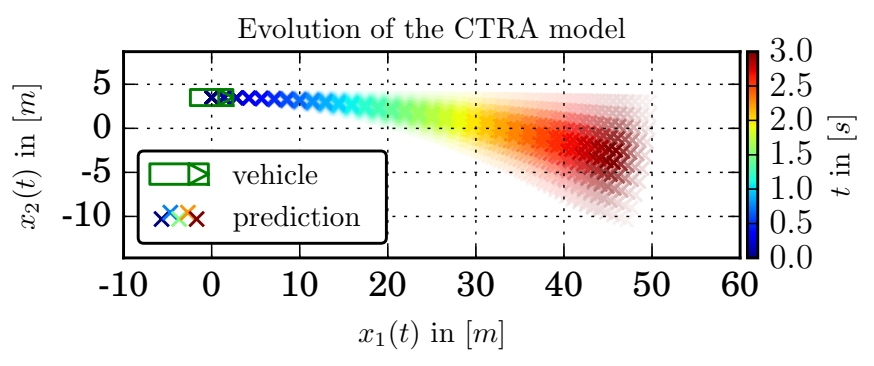

Fig. 3. Reachability of the CTRA model for the inputs from Fig. 2.

to be $3 s$ and simulated with $0.1 s$ step size. It is shown that the area of possible vehicle position widens up with farther prediction but concentrates around the case where the driver would keep the actual inputs $a_{0}$ and $\omega_{0}$. The tendency to the right is caused by the initial yaw rate being negative. After $2 s$ (yellow area) the uncertainty of lateral residence of the vehicle already widens up to more than $6 \mathrm{~m}$. This resembles the realistic distribution of possible future positions due to the use of natural input distribution and supports the following calculation of risk.

\section{Time-To-ReACT BASED Risk CAlCulation}

After the prediction of possible scene outcomes, the detection of incidents that lead to risk needs to be conducted. In the case of accidents, those incidents are collisions.

\section{A. Collision Detection}

The collision detection itself, given the state of two vehicles at a certain point in time, is calculated by determining the overlap of two vehicle's bounding boxes. Collisions of interest are between the vehicle equipped with an ADAS or $\mathrm{AD}$ function (EGO) and any other TO in the surroundings of the EGO vehicle. While the trajectories of the TOs are predicted using the introduced Monte-Carlo simulations, the future trajectory of the EGO vehicle is assumed to be certain $^{1}$. Hence only one trajectory (which is planned by the system) is considered.

A pair of trajectories can only collide once and for that reason the first occurring is registered. Continuing the example of Fig. 4, the EGO vehicle is initially placed parallel to the TO $3.5 \mathrm{~m}$ to the right with the same speed. This situation might occur, e.g., after an overtaking maneuver, where the TO wants to merge into the right lane. The results are shown in Fig. 4. Every detected collision is displayed as a pair of orange for EGO and purple vehicle for the TO. They can rarely be distinguished because $86 \%$ ( 86 out of $N_{\mathrm{smp}}=100$ ) of the possible evolutions of the TO collide with the ego vehicle.

Solely using this value as a measure of risk would be wrong, as the collisions themselves are not imminent, unavoidable or equally probable. This is indicated by the histogram on the right of the figure, where first collision

\footnotetext{
${ }^{1}$ The exact outcome of the EGO vehicle path is never completely certain due to sensor inaccuracies or control errors. However on the one hand those are small compared to the uncertainties due to driver intentions and straight forward to implement into the framework if desired on the other hand.
} 


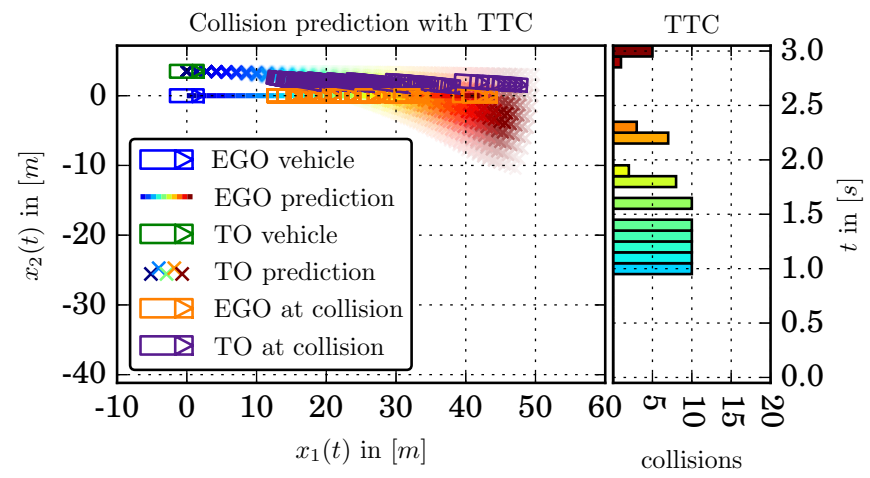

Fig. 4. Collision of the introduced traffic object with an EGO vehicle driving parallel to it.

could occur $1.0 \mathrm{~s}$ into the future while most of them will happen afterwards. The first value is equal to the worstcase TTC ${ }^{2}$ (WTTC). However, the measured TTCs and their numbers of occurrences shown in this histogram are not sufficient for the risk calculation following later. Since not every possible collision with the same temporal distance is equally risky as they are differently hard to avoid, the TTC is replaced by the time-to-react (TTR) measure in the next step.

\section{B. Time-To-React Measure}

In contrast to TTC, the TTR describes the time which is left to avoid the collision within the physical constraints of the vehicle [12]. We distinguish three possible types of evasion. Time-to-steer (TTS) is the last possible time step at which the application of full negative or positive turn rate to the EGO vehicle can avoid the collision. Full applied braking reveals the time-to-brake (TTB) value analogously. The opposite input full throttle being called time-to-kickdown (TTK). TTR is the maximum of those three considering a collision or the possible action that reveals the longest time until the necessary intervention.

The calculation of the TTR value itself is done by iterating stepwise through time, calculating paths for full left and right steering, full braking and accelerating and checking if the calculated trajectories avoid the collision. The last time step at which an evasion is possible is then saved as the TTR. If there is no collision to avoid on the currently observed trajectories, the TTR is set to infinite. Repeating this for every collision reveals a distribution of TTR over time similar to the one of TTC.

Considering the example scene again, the results are shown in Fig. 5. The constellation of the scene remains the same. Only movement prediction is faded out from the plot for better visibility of the evasion paths derived from the TTR calculations. Evasion by acceleration is not possible in this situation and hence not shown. Full braking could also avoid the collision, however it reveals less TTR and is omitted.

\footnotetext{
${ }^{2}$ The worst-case TTC (WTTC) is introduced by Wachenfeld [11]. However they used uniform sampling in the physically constrained input space in contrast to the realistic distribution from Fig. 2. Hence the worst-case time can be higher in this case.
}

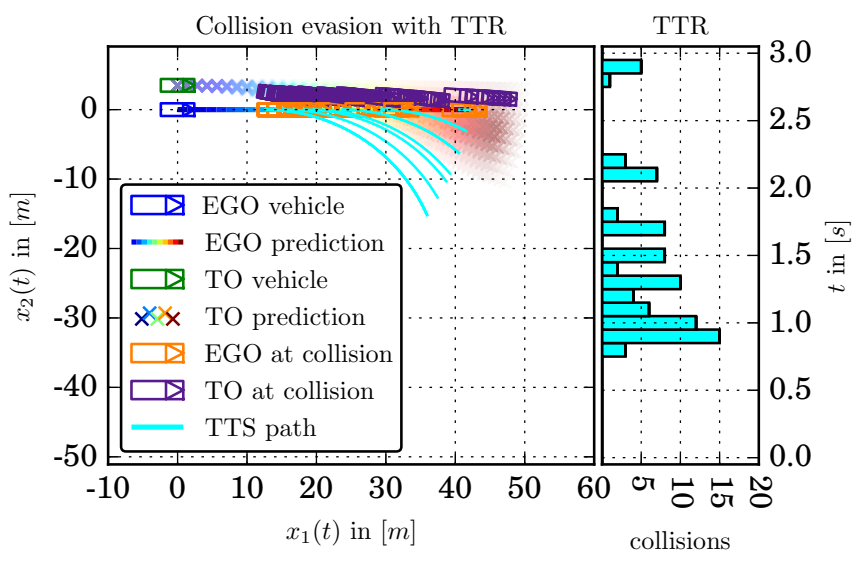

Fig. 5. The same scene as in Fig. 4 but with evasion paths of TTS shown blue. The counts of possible evasions sum up to the TTR distribution over prediction time displayed in the right histogram.

Hence full right steering is the best choice and shown by cyan paths in the plot. The histogram on the right contains the same colors referencing the type of last possible evasion. Now the shortest calculated period is $0.8 s$ until a collision is inevitable.

The TTR measure promises a better reflection of the scene risk due to the fact that it considers the possibility to avoid a collision instead of only considering the time until the impact. Hence, the following risk calculations are based on this measure and its calculated data, namely the remaining time to react per possible pair of trajectory as distribution over time and the probabilities of their occurrence.

\section{Risk Calculation}

The gathered information so far is to be processed into a single value of accident risk. It seems convenient that an imminent or even unavoidable collision should have a greater effect on such a value than one being further away and offering enough time to react. Hence, a formula is required that adds up the possible collisions weighted with a function depending on TTR and probability of occurrence.

At first, there is a certain amount of time required for an intelligent vehicle to detect the danger, plan the avoidance maneuver and initiate the actuators. This is called the point of no return $T_{\mathrm{PNR}}$ up to which regardless if an evasion is technically possible, the vehicle would not be able to perform it. Hence, all collisions with a TTR below that point are weighted $100 \%$ into the risk value. The reaction time value is of course system dependent and can not be defined globally. The next point $T_{\max }$ is defined as time from which on detected collisions are not relevant anymore. This is because the long remaining reaction times do not unveil a real risk. Between $T_{\mathrm{PNR}}$ and $T_{\max }$ a monotonic decrease of importance weighting from $100 \%$ to $0 \%$ is desired. The 


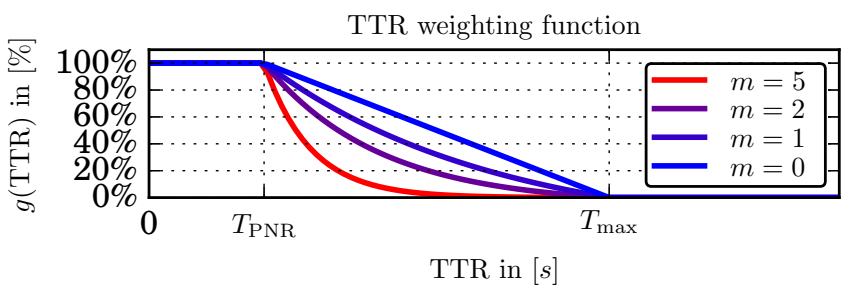

Fig. 6. The TTR weighting function from (5) plotted for steepness parameter $m=\{5,2,1,0\}$.

following function satisfies these requirements

$$
\begin{aligned}
g(\mathrm{TTR}) & =\min (1, \max (0, \bar{g}(\mathrm{TTR}))) \\
\bar{g}(\mathrm{TTR}) & = \begin{cases}\frac{e^{-m\left(\mathrm{TTR}-T_{\mathrm{PNR}}\right)}-e^{-m\left(T_{\max }-T_{\mathrm{PNR}}\right)}}{1-e^{-m\left(T_{\max }-T_{\mathrm{PNR}}\right)}} & m>0 \\
\frac{T_{\max }-\mathrm{TTR}}{T_{\max }-T_{\mathrm{PNR}}} & m=0\end{cases} \\
T_{\mathrm{PNR}} & <T_{\max }, \quad m \geq 0,
\end{aligned}
$$

where $m$ is a parameter for controlling the steepness of the monotonic decrease. This function is plotted in Fig. 6 for $m=\{5,2,1,0\}$, with $m=0$ revealing a linear decrease. Choosing the values for $m$ and $T_{\max }$ provides an opportunity to the user to tune the method, whereas $T_{\mathrm{PNR}}$ is defined by the AD system under test.

Let $\mathrm{TO}_{k}$ be the $k$ th $\mathrm{TO}$ and $\mathcal{V}_{k}$ the set of all possible feature trajectories of $\mathrm{TO}_{k}$. Then, the weighting (5) can be generalized to $g\left(\mathrm{TTR}_{k, v_{i}^{(k)}}\right)$, describing the criticality for the EGO vehicle of the $i$ th trajectory $v_{i}{ }^{(k)}$ in $\mathcal{V}_{k}$. The risk $r_{\mathrm{TO}_{k}}$ of an accident with a single traffic object $\mathrm{TO}_{k}$ is

$$
r_{\mathrm{TO}_{k}}=\sum_{i=1}^{N_{\mathrm{smp}}^{(k)}} p_{k, v_{i}^{(k)}} \cdot g\left(\mathrm{TTR}_{k, v_{i}^{(k)}}\right),
$$

where $N_{\mathrm{smp}}^{(k)}$ is the cardinality of $V_{k}$ and $p_{k, v_{i}^{(k)}}$ is the probability of $\mathrm{TO}_{k}$ following trajectory $v_{i}^{(k)}$, thus the probability of the underlying input tuple from (4). Due to the constraint target domains of $p_{k, v_{i}^{(k)}}$ and $g\left(\mathrm{TTR}_{k, v_{i}(k)}\right)$ and the weighted sum $r_{\mathrm{TO}_{k}}$ always unveils a value in the interval $[0,1]$. However due to the TTR weighting it should not be directly interpreted as collision probability, but rather as threat level.

Up to now, just the interaction with a single traffic object $\mathrm{TO}_{k}$ was considered. For further risk consolidation multiple TOs have to be taken into account by iterating over the parameter $k$.

\section{Multi-Vehicle Risk Consolidation}

Let $\mathcal{S}$ be a set similar to the one in [6] including all outcomes evolving from a given scene and taking $N_{\text {TO }}$ TOs into account. This set is spanned by

$$
\mathcal{S}=\mathcal{V}_{1} \times \mathcal{V}_{2} \times \cdots \times \mathcal{V}_{N_{\mathrm{TO}}}
$$

and contains $|\mathcal{S}|=N_{\mathrm{smp}}^{N_{\mathrm{TO}}}$ elements under the assumption that each $\mathrm{TO}_{k}$ has the same cardinality $N_{\text {smp }}$ for $V_{k}$. By design of the system the TOs are not independent from each other. An accident with at least one TO at the time step $t=t_{a}$ should prevent further accidents by the remaining TOs with the EGO for $t>t_{a}$. This kind of system design guarantees the occurrence of maximal a single accident per specific scene at $t=t_{a}$ and creates a stochastic dependence between the collision risks of individual TOs. A valid definition for the consolidated risk of this multiple vehicles system is given by

$$
\begin{aligned}
r_{\mathrm{dep}} & =\sum_{s \in \mathcal{S}} p_{s} \cdot g\left(\mathrm{TTR}_{\mathrm{acc}}\right) \\
p_{s} & =\prod_{k \in\left\{1, \ldots, N_{\mathrm{TO}}\right\}} p_{v_{s}^{(k)}} \\
\mathrm{TTR}_{\mathrm{acc}} & =\min \left(\left\{\mathrm{TTR}_{k, v_{s}^{(k)}} \mid v_{s}^{(k)} \in \mathcal{V}_{k}\right.\right. \\
\wedge k & \left.\left.\in\left\{1, \ldots, N_{\mathrm{TO}}\right\}\right\}\right),
\end{aligned}
$$

with $s$ describing a specific outcome of the scene by fixing the trajectory $i$ for each of the $k$ TOs to $v_{s}^{(k)}$. The weighted sum over the criticality of all members of $\mathcal{S}$ is still valid analogue to having a single TO in the scene. Taking the minimal TTR among all TOs in a specific outcome defines the risk of the outcome. The TTR values for a certain TO can be calculated independent from the other TOs. This fact rises from (8) as in case of a collision the accident is unique and the minimal TTR is exactly the TTR of an TO having an accident at $t=t_{a}$. To prove the latter one has to examine in detail the only two types of TOs existing which differ from their corresponding independent TTR value. The first are described by TOs causing an accident with the EGO in the independent case but non in the dependent case because another TO is already collided with the EGO. Therefore, its TTR becomes infinite. The second type is collision free in the independent case but its trajectory crosses the location of the accident at some time step $t>t_{a}$ in the dependent case. Again the TTR in this case is guaranteed to be larger than for the TTR of a TO involved in the unique accident. In addition, the case in the model is labeled not an accident as it happens later than the unique accident at $t=t_{a}$ and is irrelevant in terms of risk assessment. Being the reason for the rising dependencies, it is obvious that if no accident occurs at all there will be no differences as all TOs will have an infinite TTR and contribute according to the weight function in (5) with zero risk. Thus, taking each case into consideration the minimal TTR is unambiguous regardless of independence or dependence and refers to a TO causing the unique accident at the time step $t=t_{a}$.

Although saving computational costs by proving that it is sufficient to deploy the independent TTR values for the multi-vehicle risk, (8) has still an exponential computational complexity $\mathcal{O}\left(N_{\mathrm{smp}}^{N_{\mathrm{TO}}}\right)$. A computational less expensive alternative offers the approximation of $r_{i n d}$ by the independent risk

$$
r_{\text {ind }}=1-\prod_{k=1}^{N_{\mathrm{TO}}}\left(1-r_{\mathrm{TO}_{k}}\right),
$$

calculated by its complement meaning the probability that no accident occurs in the complete scene. In contrast to 
$r_{d e p}$, the independent risk calculation via (9) has just a linear complexity $\mathcal{O}\left(N_{\mathrm{smp}} \cdot N_{\mathrm{TO}}\right)$ with respect to the number of TOs $N_{\text {TO }}$ in the scene. In addition, TOs which do not collide with the EGO vehicle within $T_{\max }$ can be excluded as their TTRs are infinite and as mentioned before will either be neglected by the minimum function in (8) or contribute with zero impact on the risk. Last but not least, it is claimed that the independent calculated risk $r_{\text {ind }}$ is always an overestimation to the dependent risk $r_{\text {dep }}$ and therefore satisfies the requirements on the method. If a critical threshold $r_{\text {thresh }}$ of risk is exceeded by an independent calculation (9), one can still apply the dependent formula (8) to revise the result. A proof for the overestimation is given below.

Proof: Assuming independency overestimates the risk. Let $\mathcal{A}_{k}^{(s)}$ be the event of having an accident with $\mathrm{TO}_{k}$ in a specific scenario $s \in \mathcal{S}$ and $\mathcal{P}\left(\mathcal{A}_{k}^{(s)}\right)$ the corresponding risk of the event

$$
\mathcal{P}\left(\mathcal{A}_{k}^{(s)}\right)=g\left(\mathrm{TTR}_{k, v_{s}^{(k)}}\right) .
$$

For clarity the following substitution is defined:

$$
r_{k, v_{i}^{(k)}}=p_{k, v_{i}^{(k)}} \cdot g\left(\mathrm{TTR}_{k, v_{i}^{(k)}}\right) .
$$

By inserting (6) in (9) and regrouping the terms, the independent risk $r_{\text {ind }}$ can be expressed as sum over the scene space $\mathcal{S}$.

$$
\begin{aligned}
& r_{\mathrm{ind}}=1-\prod_{k=1}^{N_{\mathrm{TO}}}\left(1-\sum_{i=1}^{N_{\mathrm{smp}}^{(k)}} r_{k, v_{i}^{(k)}}\right) \\
& =\sum_{k \in\left\{1, \ldots, N_{\mathrm{TO}}\right\}} r_{k, v_{i}^{(k)}}-\sum_{k_{1} \neq k_{2}} r_{k_{1}, v_{i}^{\left(k_{1}\right)}} \cdot r_{k_{2}, v_{i}^{\left(k_{2}\right)}}+\ldots \\
& i \in\left\{1, \ldots, N_{\mathrm{smp}}\right\} \quad k_{1}, k_{2} \in\left\{1, \ldots, N_{\mathrm{TO}}\right\} \\
& i_{1}, i_{2} \in\left\{1, \ldots, N_{\mathrm{smp}}\right\}
\end{aligned}
$$

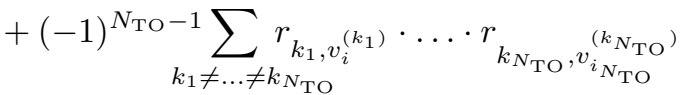

$$
\begin{aligned}
& k_{1}, \cdots, k_{N_{\mathrm{TO}}} \in\left\{1, \ldots, N_{\mathrm{TO}}\right\} \\
& i_{1}, \cdots, i_{N_{\mathrm{TO}}} \in\left\{1, \ldots, N_{\mathrm{smp}}\right\} \\
& =\sum_{s \in \mathcal{S}} p_{s}\left[\sum_{k \in\left\{1, \ldots, N_{\mathrm{TO}}\right\}} \mathcal{P}\left(\mathcal{A}_{k}^{(s)}\right)-\sum_{\substack{k_{1} \neq k_{2} \\
k_{1}, k_{2} \in\left\{1, \ldots, N_{\mathrm{TO}}\right\}}} \mathcal{P}\left(\mathcal{A}_{k_{1}}^{(s)}\right) \cdot \mathcal{P}\left(\mathcal{A}_{k_{2}}^{(s)}\right)\right. \\
& \left.+\cdots+(-1)^{N_{\mathrm{TO}}-1} \cdot \prod_{k=1}^{N_{\mathrm{TO}}} \mathcal{P}\left(\mathcal{A}_{k}^{(s)}\right)\right] \\
& =\sum_{s \in \mathcal{S}} p_{s} \cdot \mathcal{P}_{\text {ind }}\left(\mathcal{A}_{1}^{(s)} \cup \ldots \cup \mathcal{A}_{N_{\mathrm{TO}}}^{(s)}\right)
\end{aligned}
$$

The result is in accordance with the original design and represents as expected the independent risk as the union of all individual TO risks per scenario $s$ weighted by $p_{s}$ as a scenario specific occurrence factor.

Similar, the dependent risk (8) can be expressed,

$$
r_{\text {dep }}=\sum_{s \in S} p_{s} \cdot g\left(\mathrm{TTR}_{\mathrm{acc}}\right)=\sum_{s \in S} p_{s} \cdot \mathcal{P}_{\mathrm{dep}}\left(\mathcal{A}_{\mathrm{acc}}^{(s)}\right),
$$

with $\mathcal{A}_{\text {acc }}$ is the event of the unique accident in the dependent case and $\mathcal{P}\left(\mathcal{A}_{\text {acc }}\right)$ is the corresponding occurrence probability.
Equation (12) and (13) can be compared scenario by scenario. However, one has to distinguish between two types of scenarios. The first type represents an accident free scenario $s^{\prime}$ in $\mathcal{S}^{\prime}$ for each TO concerning the EGO. As already mentioned, this kind of scenario will contribute neither in the independent nor in the dependent model to the risk value.

$$
\mathcal{P}_{\text {dep }}\left(\mathcal{A}_{\text {acc }}^{\left(s^{\prime}\right)}\right)=\mathcal{P}_{\text {ind }}\left(\mathcal{A}_{1}^{\left(s^{\prime}\right)} \cup \ldots \cup \mathcal{A}_{N_{\text {TO }}}^{\left(s^{\prime}\right)}\right)=0 \quad \forall s^{\prime} \in \mathcal{S}^{\prime}
$$

The second type includes all remaining scenarios $s^{\prime \prime} \in \mathcal{S}^{\prime \prime}$, containing at least one accident in the independent model meaning an unique accident in the dependent model. If only a single accident of one TO with the EGO occurs in the independent model the scenario is identical to the dependent model. For more than one accident in the independent case, the contribution of the scenario to the risk value is defined by the joined risk of all TOs having an accident according to equation (12). As the scenario in the dependent model doesn't differ until the unique accident happens, the risk term of the TO causing the unique accident has to be a part of the independent risk consideration.

$$
\mathcal{A}_{\mathrm{acc}}^{\left(s^{\prime \prime}\right)} \subseteq \mathcal{A}_{1}^{\left(s^{\prime \prime}\right)} \cup \ldots \cup \mathcal{A}_{N_{\mathrm{TO}}}^{\left(s^{\prime \prime}\right)} \quad \forall s^{\prime \prime} \in \mathcal{S}^{\prime \prime}
$$

Therefore, the risk per scenario in the independent model is estimated regardless of the scenario type equal or higher as in the dependent model.

$$
\mathcal{P}_{\text {dep }}\left(\mathcal{A}_{\text {acc }}^{(s)}\right) \leq \mathcal{P}_{\text {ind }}\left(\mathcal{A}_{1}^{(s)} \cup \ldots \cup \mathcal{A}_{N_{\mathrm{TO}}}^{(s)}\right) \quad \forall s \in \mathcal{S}
$$

Taking the equality of $p_{s}$ in both models and the summation over all scenarios $s$ in consideration, yields the final proof for the risk overestimation by choosing the independent model.

$$
r_{\text {dep }} \leq r_{\text {ind }}
$$

\section{EXPERIMENTAL RESULTS}

The following evaluations on representative scenarios use all the same method parametrization listed in Table II. The reason for the chosen prediction time of $1 s$ longer than the maximum risk time is that predicted collisions occurring between those values can have a TTR below $2 s$ and hence still affect the risk value.

First two simple simulative real world experiments verify the new measure against expectations and compare it with common simple threat indicators. Following a more complex real world experiment featuring two TOs shows the applicability to real driving data.

TABLE II

PARAMETRIZATION OF THE METHOD FOR EVALUATION

\begin{tabular}{lcc} 
Name & Symbol & Value \\
\hline \hline prediction horizon & - & $3.0 s$ \\
prediction step & - & $0.1 \mathrm{~s}$ \\
trajectories per TO & $N_{\mathrm{smp}}$ & 100 \\
point of no return & $T_{\mathrm{PNR}}$ & $0.5 \mathrm{~s}$ \\
maximum risk time & $T_{\max }$ & $2.0 \mathrm{~s}$ \\
weighting slope & $m$ & 1.0 \\
consolidation threshold & $t_{\text {thresh }}$ & $10 \%$
\end{tabular}


Risk of a simulated rear end collision

$$
x_{1}(t) \text { in }[m]
$$

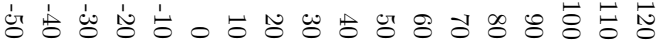

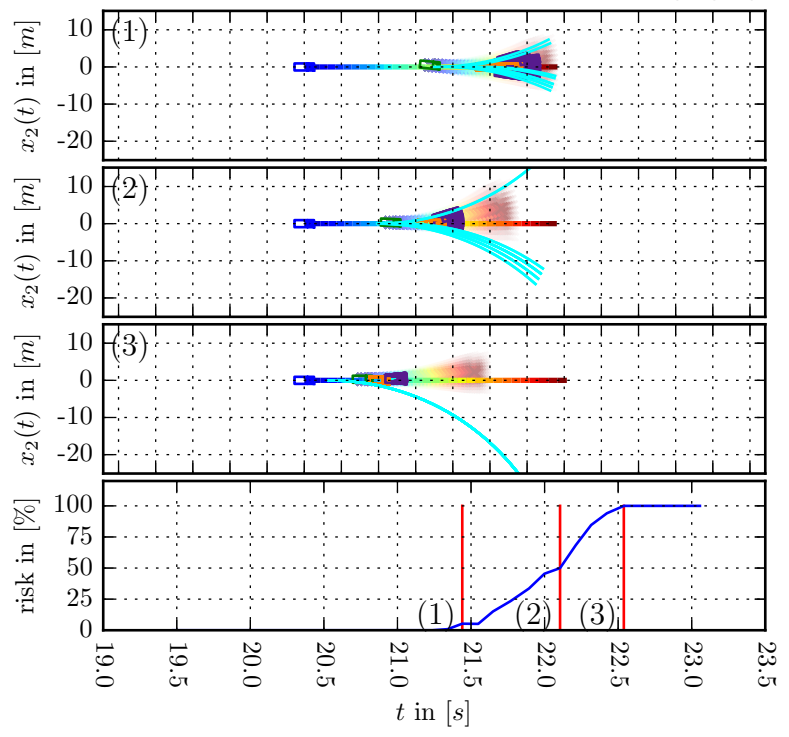

Fig. 7. Calculated risk of a simulated rear end collision scenario.

\section{A. Simulative Experiments}

A first expectation on a risk measure would be a $100 \%$ amplitude on a scenario with an actual or unavoidable accident. Additionally, a monotonic increase of risk up to the collision is desired. For this test a scenario where the EGO vehicle driving $21.0 \frac{\mathrm{m}}{\mathrm{s}}$ collides with the rear of a TO driving $10.0 \frac{\mathrm{m}}{\mathrm{s}}$ is simulated. Three scenes and the temporal development of the risk measure are shown in Fig. 7. For better comparability of the scenes this and all following experiment plots are shown in EGO vehicle fixed coordinates. Snapshot (1) reveals a scene where first possible collisions are detected, but large reaction times reveal very little risk. In scene (2) the TTRs are much lower and a reasonable risk of $\sim 50 \%$ is measured. In (3) the collision is unavoidable with respect to the $0.5 \mathrm{~s}$ point of no return. Both expectations, the monotonic increase and the $100 \%$ amplitude are met.

In a second experiment an EGO vehicle driving $26.0 \frac{\mathrm{m}}{\mathrm{s}}$ bypassing a TO at half the velocity is simulated and shown in Fig. 8. Again first collisions are detected in scene (1) but provide plenty of time for reaction. An insignificant peak in risk is obtained in (2) by collisions with TTR below $2.0 \mathrm{~s}$ on improbable TO trajectories. At scene (3) the EGO vehicle is close enough to the TO that a lateral movement within the bounds of realistic driving behavior cannot cause a collision anymore. In reality, such a bypass scenario is common on highways and considered safe. Again the expectations are met.

The calculated risk measure for both experiments is again shown in Fig. 9. The upper plot shows the risk of the rear end scenario compared to the TTC, WTTC and headway time (THW). All of the latter (TTX) are measured in seconds and hence more critical the closer to zero they are. They all converge linearly towards $0 s$ up to the actual collision

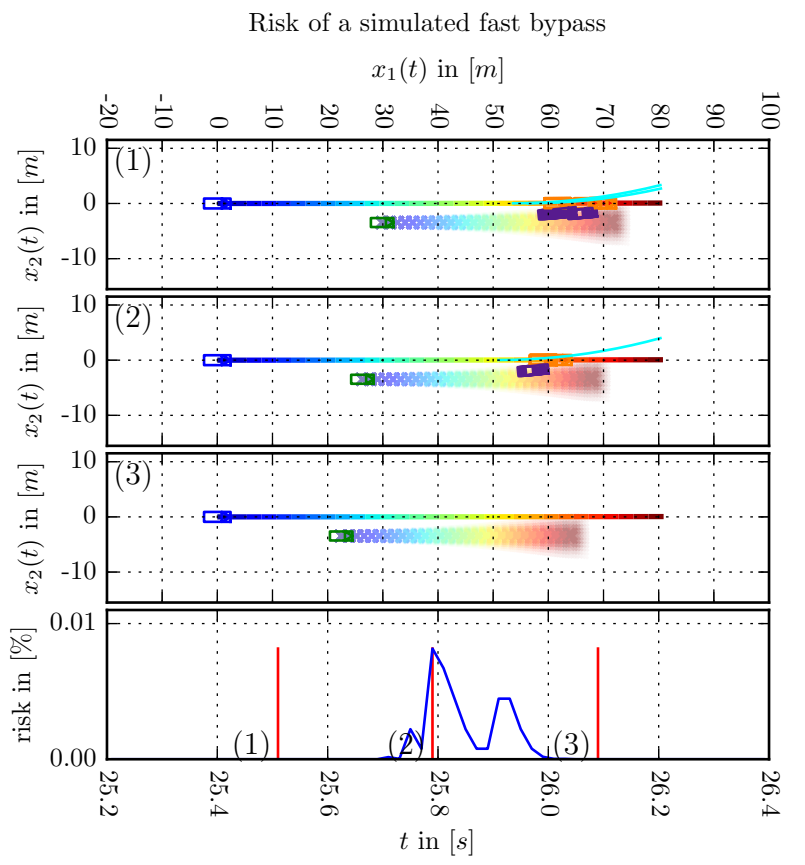

Fig. 8. Calculated risk of a simulated passing by scenario with high relative velocity.

Comparison of risk measure with common TTX
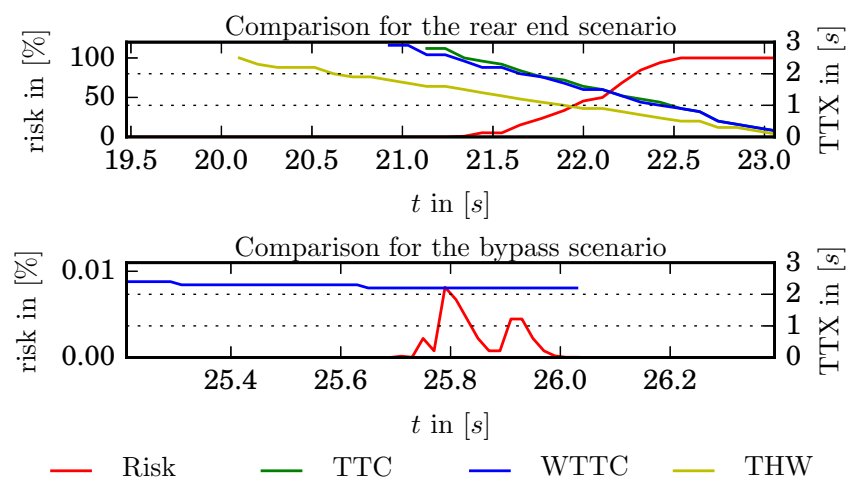

Fig. 9. Comparsion of the risk measure with TTC, WTTC and THW (grouped as TTX) for the rear end collision (top) and the bypass scenario (bottom).

and neglect the reaction time of the vehicle. Whereas the proposed risk measure reveals the highest risk earlier and shows a steeper ascent contemplating reaction times. In the lower plot the risk of the bypass is compared to common threat indicators. TTC and THW are not calculable because there is no collision on the main pathway of the vehicles. The WTTC reveals approximately the same risk until the vehicles cannot collide anymore. It assigns the same risk to the bypass as in the rear end scenario $1.5 \mathrm{~s}$ before the collision, which seems to be exaggerated.

\section{B. Real world experiment involving two vehicles}

The last experiment is conducted on a real world measure from a German highway. The EGO vehicle follows another TO at $\sim 26.0 \frac{m}{s}$. In (1) a second TO is driving in parallel to 


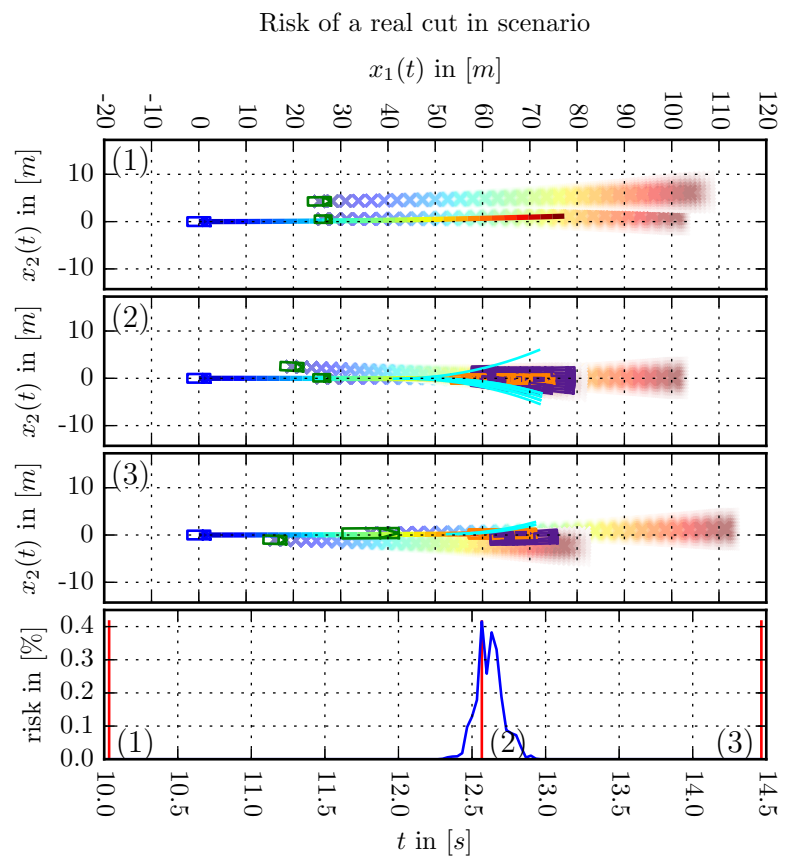

Fig. 10. Calculated risk of a real cut in scenario involving two TOs.

the first in the left lane until it decelerates and merges into the safety clearance of the EGO vehicle in the right lane in (2). A peak of $\sim 0.4 \%$ risk at this point arises due to the approach of the cutting in TO to the EGO. The latter immediately decelerates in (3) to $\sim 24.0 \frac{\mathrm{m}}{\mathrm{s}}$ to regain safety clearance and the risk vanishes. The assessment method assigned low risk to the proper reaction of the EGO vehicle in this scenario.

\section{CONCLusion ANd Future Work}

A novel risk assessment method fitted to the requirements of testing $\mathrm{AD}$ is proposed. In order to depict a realistic measure, a Monte-Carlo prediction for possible future TO paths in parameterized with naturalistic driving data is developed. The basis of a single paths threat is based on the TTR measure to reflect the capabilities of the EGO vehicle avoiding the collisions. Stochastic consolidation of gathered information into a single scene risk value between 0 and 1 is derived. To overcome the infeasible computational load resulting from crowded scenes, an over-approximation of the risk is suggested and mathematically proven to comply with the requirement of avoiding false negatives. Finally simulative and a real road test show that expectations are met and the proposed risk value reveals more comprehensive measures than common simple threat indicators.

Within this work a simple vehicle model for prediction is used under the assumption of suitability for short prediction times is used. Interesting future research would examine the effect of more complex, maneuver-based or even interaction aware models on this risk measure. Furthermore, perceived subjective risk by the occupant can be coded into the risk value and compared to the physical risk of this work.

\section{ACKNOWLEDGMENT}

The authors gratefully acknowledge the financial support by the BMW Group within the CAR@TUM project.

\section{REFERENCES}

[1] H. Winner and W. Wachenfeld, "Validation of Automated Driving Strategies and Challanges," Aschaffenburg, May 2016.

[2] F. Schuldt, F. Saust, B. Lichte, M. Maurer, and S. Scholz, "Effiziente systematische Testgenerierung fÃCEr Fahrerassistenzsysteme in virtuellen Umgebungen," Automatisierungssysteme, Assistenzsysteme und Eingebettete Systeme FÃCr Transportmittel, 2013.

[3] L. Eckstein and A. Zlocki, "Safety potential of ADASâCombined methods for an effective evaluation," in 23rd International Technical Conference on the Enhanced Safety of Vehicles (ESV) Seoul, South Korea, 2013.

[4] M. Tatar and J. Mauss, "Systematic test and validation of complex embedded systems," ERTS-2014, Toulouse, pp. 05-07.02, 2014.

[5] K. Groh, T. Kuehbeck, M. Schiementz, and C. Chibelushi, "Towards a Scenario-Based Assessment Method for Highly Automated Driving Functions," in 8th Conference on Driver Assistance, Munich, Germany, Sep. 2017.

[6] A. Lawitzky, D. Althoff, C. F. Passenberg, G. Tanzmeister, D. Wollherr, and M. Buss, "Interactive scene prediction for automotive applications," in Intelligent Vehicles Symposium (IV), 2013 IEEE. IEEE, 2013, pp. 1028-1033.

[7] S. Ammoun and F. Nashashibi, "Real time trajectory prediction for collision risk estimation between vehicles," in Intelligent Computer Communication and Processing, 2009. ICCP 2009. IEEE 5th International Conference on. IEEE, 2009, pp. 417-422.

[8] A. Tamke, T. Dang, and G. Breuel, "A flexible method for criticality assessment in driver assistance systems," in Intelligent Vehicles Symposium (IV), 2011 IEEE. IEEE, 2011, pp. 697-702.

[9] A. Polychronopoulos, M. Tsogas, A. Amditis, U. Scheunert, L. Andreone, and F. Tango, "Dynamic situation and threat assessment for collision warning systems: The EUCLIDE approach," in Intelligent Vehicles Symposium, 2004 IEEE. IEEE, 2004, pp. 636-641.

[10] M. Althoff and A. Mergel, "Comparison of Markov Chain Abstraction and Monte Carlo Simulation for the Safety Assessment of Autonomous Cars," IEEE Transactions on Intelligent Transportation Systems, vol. 12, no. 4, pp. 1237-1247, 2011.

[11] W. Wachenfeld, P. Junietz, R. Wenzel, and H. Winner, "The worst-timeto-collision metric for situation identification," in 2016 IEEE Intelligent Vehicles Symposium (IV), Jun. 2016, pp. 729-734.

[12] J. Hillenbrand, A. M. Spieker, and K. Kroschel, "A multilevel collision mitigation approachâlts situation assessment, decision making, and performance tradeoffs," IEEE Transactions on intelligent transportation systems, vol. 7, no. 4, pp. 528-540, 2006.

[13] S. Lef Ãšvre, D. Vasquez, and C. Laugier, "A survey on motion prediction and risk assessment for intelligent vehicles," ROBOMECH Journal, vol. 1, no. 1, p. 1, Jul. 2014.

[14] A. Broadhurst, S. Baker, and T. Kanade, "Monte Carlo road safety reasoning," in Intelligent Vehicles Symposium, 2005. Proceedings. IEEE. IEEE, 2005, pp. 319-324.

[15] A. Eidehall and L. Petersson, "Statistical threat assessment for general road scenes using Monte Carlo sampling," IEEE Transactions on intelligent transportation systems, vol. 9, no. 1, pp. 137-147, 2008.

[16] S. Ulbrich, T. Menzel, A. Reschka, F. Schuldt, and M. Maurer, "Defining and substantiating the terms scene, situation, and scenario for automated driving," in Intelligent Transportation Systems (ITSC), 2015 IEEE 18th International Conference on. IEEE, 2015, pp. 982-988.

[17] M. Brannstrom, E. Coelingh, and J. Sjoberg, "Model-based threat assessment for avoiding arbitrary vehicle collisions," IEEE Transactions on Intelligent Transportation Systems, vol. 11, no. 3, pp. 658-669, 2010.

[18] P. Lytrivis, G. Thomaidis, and A. Amditis, "Cooperative path prediction in vehicular environments," in Intelligent Transportation Systems, 2008. ITSC 2008. 11th International IEEE Conference on. IEEE, 2008, pp. 803-808.

[19] M. Althoff, O. Stursberg, and M. Buss, "Erreichbarkeitsanalyse von Verkehrsteilnehmern zur Verbesserung von Fahrerassistenzsystemen," in Proc. of 3. Tagung Aktive Sicherheit durch Fahrerassistenz, 2008.

[20] C. Kessler and A. Etemad, "SP 6 D6.8 FOT Data," Jun. 2012. 